\title{
Usefulness of Hybrid Surgery Combining Original CEA and CAS for Carotid Artery Stenosis with a Large Amount of Vulnerable Plaque
}

Yoshinobu Sekihara, Yuzo Saito, Yoshiyuki Takada, and Norihiro Ishii

Objective: For carotid artery stenosis with a large amount of vulnerable plaque in a wide range, we performed a hybrid surgery combining carotid endarterectomy (CEA) and carotid artery stenting (CAS), and report the results of treatment. Methods: Surgical treatment for carotid artery stenosis in 216 patients was performed between January 2016 and June 2018. Of these, 15 patients were treated in a hybrid operating room because both CEA and CAS were judged to be risky. We treated these patients with preparation of stenting for remote lesions far from the CEA arterial incision. The perioperative treatment results were retrospectively examined.

Results: Of the 15 patients treated in a hybrid operating room, 10 were stented after CEA. All these cases were treated by retrograde stent placement in the proximal common carotid artery (CCA). Treatment was completed in all patients, and no cerebral infarction, myocardial infarction, or death was observed in the perioperative period. There were no cases of additional neurological events during the follow-up period, but asymptomatic restenosis was observed in one patient. Conclusion: Hybrid surgery combining CEA and CAS was considered to be an effective treatment for carotid artery stenosis with a large amount of vulnerable plaque.

Keywords $>$ carotid artery stenosis, hybrid surgery, carotid endarterectomy, carotid artery stenting

\section{Introduction}

The efficacy of carotid endarterectomy (CEA) for carotid artery stenosis has been established, ${ }^{1,2)}$ but it is difficult to secure a surgical field in patients with high-level lesions in which plaque involves the $\mathrm{C} 2$ vertebral body to cranial side or low-level lesions in which plaque involves the $\mathrm{C} 6$ vertebral body to aortic side; therefore, carotid artery stenting (CAS) is selected in many cases. ${ }^{3)}$ However, when the greater portion of large-volume plaque involving an extensive area is expected to be vulnerable plaque consisting of hemorrhage and lipid components, CAS is not appropriate due to the risk of distal embolism. $\left.{ }^{4}\right)$ At our institute,

Department of Neurosurgery, New Tokyo Hospital, Matsudo, Chiba, Japan

Received: December 27, 2019; Accepted: October 21, 2020 Corresponding author: Yoshinobu Sekihara. Department of Neurosurgery, New Tokyo Hospital, 1271, Wanagaya, Matsudo, Chiba 270-2232, Japan

Email: nougesekihara@gmail.com

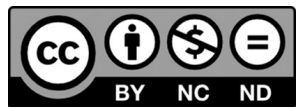

This work is licensed under a Creative Commons Attribution-NonCommercialNoDerivatives International License.

(C)2021 The Japanese Society for Neuroendovascular Therapy combined CEA+CAS has been performed on high-risk patients regarding both CEA and CAS in the hybrid operating room. We report the results of treatment.

\section{Materials and Methods}

Surgical treatment for carotid artery stenosis was performed on 216 patients between January 2016 and June 2018. Among them, the 15 subjects were treated in the hybrid surgery room due to the risks of both CEA and CAS.

Asymptomatic patients, those with $\geq 80 \%$ stenosis on assessment using the NASCET method, were treated. Symptomatic patients, those with $\geq 50 \%$ stenosis on assessment using the same method, were treated. However, surgery was indicated for some patients with vulnerable plaque as the source of embolism even when the percent stenosis was low.

\section{Selection of treatment}

For patients with cervical internal carotid artery (ICA) stenosis, carotid ultrasonography and black blood magnetic resonance imaging (BB-MRI) were conducted before surgery, and surgical procedures were determined by evaluating the properties, volume, and extent of plaque. At our institute, vulnerable plaque was defined as exhibiting low-level 
brightness in the greater portion on carotid ultrasonography and having a large amount of hemorrhage or lipid components with a signal intensity higher than that of the sternocleidomastoid muscle on T1- and T2-weighted BB-MRI. Furthermore, plaque with ulcer formation or mobility was also regarded as vulnerable. In the above patients with vulnerable plaque for whom general anesthesia was possible, CEA was selected. At our institute, CEA has been adopted as a first-choice treatment. In patients with hard plaque primarily consisting of fiber components, those for whom general anesthesia was difficult due to a poor general condition, and those with recurrence following CEA, CAS has been selected.

For patients with extensive, large-volume, vulnerable plaque, which is difficult to treat by CEA, and those for whom safe treatment by CEA or CAS alone was considered to be difficult due to tandem lesions, which are present at distant sites, such as the internal-external carotid bifurcation and proximal common carotid artery (CCA) or brachiocephalic artery, hybrid CEA+CAS surgery, involving preparations for additional stenting in the hybrid operating room where a single-plane angiograph (Philips, Amsterdam, Netherlands) was installed, has been selected.

\section{Procedures of hybrid surgery}

Under general anesthesia, CEA was initially performed while conducting cerebral oxygen saturation monitoring with near-infrared ray and somatosensory evoked potential (SEP)/motor evoked potential (MEP) monitoring. The anterior cervical skin along with the anterior margin of the sternocleidomastoid muscle was incised to expose three cervical branches and secure them with vascular tape. After arteriotomy through transient blockage, plaque at the internal-external carotid bifurcation was removed. Subsequently, an 8-F Launcher (Medtronic, Minneapolis, MN, USA) or 6-F sheath was retrogradely inserted through the site of arteriotomy, and stenting was performed (Fig. 1).

In general, dual antiplatelet therapy (DAPT) using biaspirin at $100 \mathrm{mg}$ and clopidogrel at $75 \mathrm{mg}$ was performed. Heparin at 3000-5000 units was intravenously injected before arterial blockage for CEA to confirm that the activated clotting time (ACT) was prolonged by approximately two-fold before intravenous injection. On the day of surgery, the presence of neurological symptoms/wound swelling was examined in addition to blood pressure control in the intensive care unit (ICU). After surgery, regular follow-up imaging was performed using carotid ultrasonography and MRI. For patients in whom restenosis was suspected, computed tomography (CT) angiography or angiography was conducted.

\section{Investigation items}

We retrospectively examined the perioperative results of treatment in patients who had undergone hybrid surgery for carotid artery stenosis. The patient background, contents of the procedure, success rate, perioperative cerebral infarction, incidence of myocardial infarction, death, or other complications, and incidence of restenosis were investigated.

\section{A representative case (Case 2)}

A 76-year-old man. Progressive left carotid artery stenosis was observed, although it was asymptomatic. A large volume of vulnerable plaque was continuous from the internal-external carotid bifurcation to the aortic side CCA (Fig. 2A). Under general anesthesia, the CCA involving the aortic side was exposed (Fig. 2B) and left CEA was performed. However, central-side plaque was unable to be completely removed, and a large volume of vulnerable plaque was exposed from the stump (Fig. 2C). A stent was retrogradely inserted into the CCA through the site of arteriotomy. On the side of the CCA, tourniquet blockage with vascular tape was performed. While visually inspecting the plaque stump and confirming the true lumen, a 0.035-inch guidewire was guided to the aortic arch under fluoroscopy. Using Goretex CV-6, the CCA was sutured at a distance of approximately $4 \mathrm{~cm}$ from the aortic side. To prevent massive hemorrhage on guiding-catheter insertion, two tourniquets were added. Under fluoroscopy, an 8-F Launcher was retrogradely inserted into the CCA using a 0.035-inch guidewire as guide (Fig. 2D). Left common carotid angiography was performed using a Simmons-type catheter through a 4-F sheath that had been placed in the left brachial artery (Fig. 2E). A brain-protecting sheet with a radiopaque line was placed on the dorsal surface of the CCA as a marker of the plaque stump, and used as a mark for stenting. The position of the stent was determined by referencing common carotid angiography, and the guiding catheter was pulled to a sufficient point to adjust the position. As stents, Carotid Wallstent $10 \times 31 \mathrm{~mm}$ and $10 \times 24 \mathrm{~mm}$ (Boston Scientific, Natick, MA, USA) were inserted from the aortic side to duplicate a portion (Fig. 2F). The distal end of the partially duplicated stents was inserted in order to be distal to the plaque stump, and the plaque stump was covered with the stents. Subsequently, the stents were dilated using a Mustang $9.0 \times 40 \mathrm{~mm}$ (Boston Scientific), and imagining stent crimping to the vascular wall was 


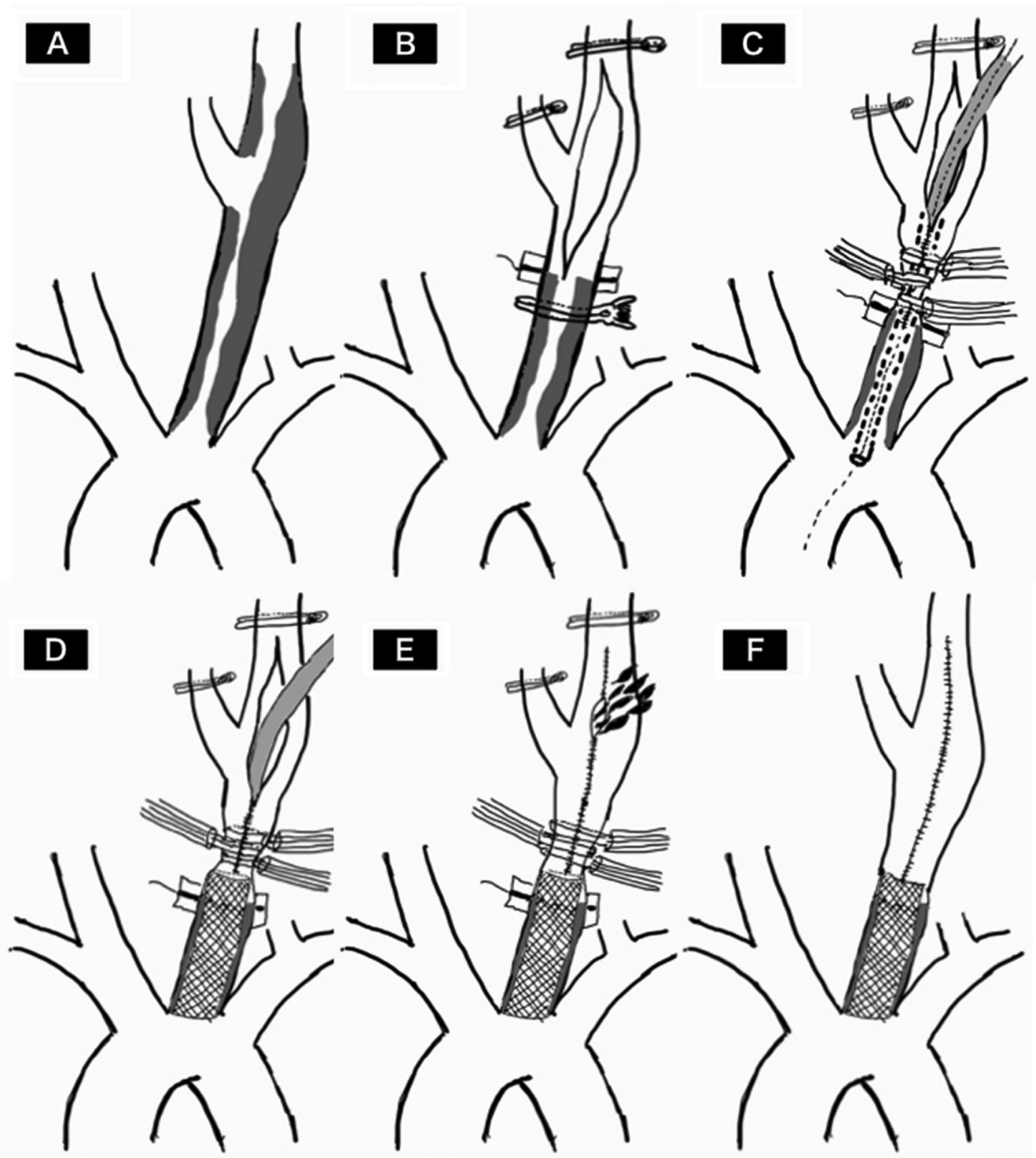

Fig. 1 An illustration of hybrid surgery combining CEA with CAS at our institute. (A) Extensive continuous plaque from ICA to CCA. (B) After removing plaque as much as possible, a brain protection surgical sheet with X-ray impermeable markers was laid on the back of the CCA to mark the plaque stump. (C) After suturing the arterial wall to some extent, three tourniquets were deployed. The guiding catheter was inserted toward aortic arch in retrograde fashion in the proximal CCA. (D) Stent placed in the proximal CCA. (E) After removing the guiding catheter, debris and thrombus were flushed through the carotid bulb. (F) Arterial sutures were completed to finish the procedure. CAS: carotid artery stenting; CEA: carotid endarterectomy; CCA: common carotid artery; ICA: internal carotid artery

carried out instead of stenotic-site extension with a balloon. While controlling the volume of blood loss by loosening the tourniquets, in-stent blood containing debris was sufficiently eliminated through the site of arteriotomy. Approximately $20-30 \mathrm{~mL}$ of blood was excreted over time. The blood retained in the surgical field was aspirated using an injector and excreted on gauze to confirm the presence of debris. We considered the contaminated blood to have been eliminated by extravasating blood containing debris retaining in the CCA from the site of arteriotomy under high pressure. The ICA was sutured, and declamping was conducted in the order of the external carotid artery, CCA, and ICA according to standard CEA procedures (Fig. 2H). Final angiography was performed to confirm the in-stent state, and the procedure was completed (Fig. 2G). Before surgery, DAPT using biaspirin at $100 \mathrm{mg}$ and clopidogrel at $75 \mathrm{mg}$ was performed. Heparin at 3000 units was intravenously injected before arterial blockage for CEA in order for ACT to be prolonged by $\geq$ two-fold compared with that before administration. After surgery, it was spontaneously 


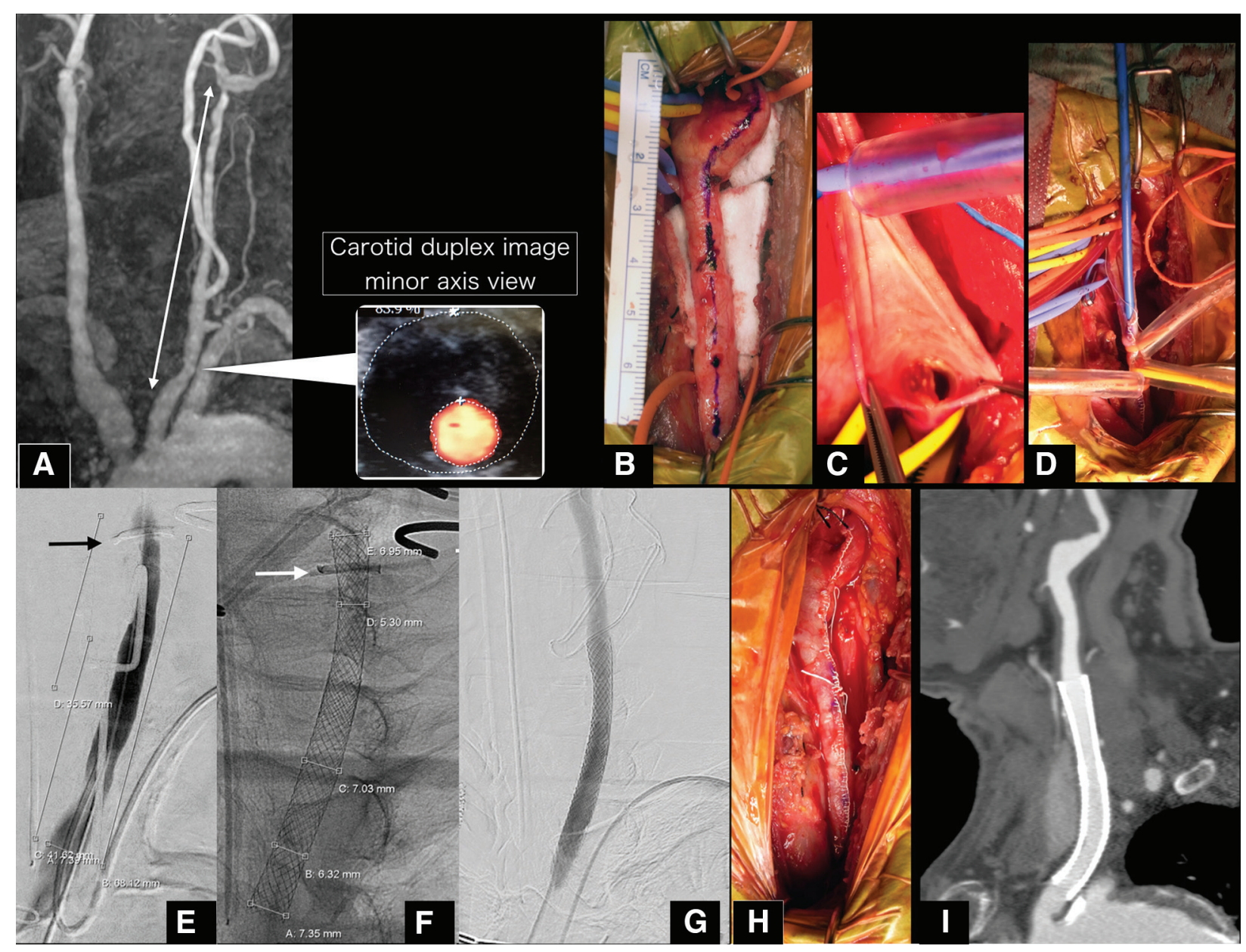

Fig. 2 Case presentation (Case 2). (A) MRA findings: A large amount of plaque continuing from the ICA to CCA. Carotid duplex image minor axis view showing a large volume of weakly enhanced plaque. (B) Intraoperative picture. We extended the operative field as long as possible. (C) A large amount of vulnerable plaque remains on the plaque stump. (D) After suturing the arterial wall, three tourniquets were deployed. A guiding catheter was inserted toward the aortic arch in a retrograde manner in the proximal CCA. (E) Preoperative angiography through a 4-F Simmons catheter. Black arrow; A brain protection surgery sheet with X-ray impermeable markers was laid on the back of the CCA to mark the plaque stump. (F) A Carotid Wallstent was deployed $(10 \times 31 \mathrm{~mm}$ and $10 \times 24 \mathrm{~mm})$. White arrow; same as the black arrow. (G) Postoperative angiography. A Carotid Wallstent was deployed. (H) Arterial suturing was completed to finish the procedure. (I) Postoperative CT angiography 3.5 years later. There was no restenosis. CCA: common carotid artery; CT: computed tomography; ICA: internal carotid artery; MRA: magnetic resonance imaging neutralized. There was no postoperative hemorrhagic complication or perioperative cerebral/myocardial infarction. The course has been favorable. CT angiography 2 years and 6 months after surgery revealed no significant restenosis (Fig. 2I). There has been no neurological event.

\section{Results}

Of the 216 patients treated during the survey period, 164 patients $(75.9 \%)$ were treated with CEA alone and 42 patients (19.4\%) were treated CAS alone. Considering the risk of a single procedure, $15(6.9 \%)$ were treated in the hybrid operating room (Table 1). They consisted of 11 males and 4 females, ranging from 43 to 82 years in age (mean: 70.9 years, median: 73.3 years). Seven patients had symptomatic lesions: transient ischemic attack (TIA) in two and cerebral infarction in five patients. The other eight patients had asymptomatic lesions. Of the 15 patients, plaque was continuous from the internal-external carotid bifurcation to the proximal CCA in nine, tandem lesions were present at the internal-external carotid bifurcation and proximal area of the CCA in two, and plaque was continuous from this bifurcation to the distal ICA in four patients. Initially, CEA was performed under general anesthesia. Subsequently, stenting was conducted in 10 patients (4.6\%); in all patients, a stent was retrogradely inserted into the proximal CCA. In eight patients, retrograde stenting involving the stump for plaque on the CCA side, which 
was unable to be fully treated by CEA, was performed. Complete occlusion at the origin of the left CCA with repeated TIAs was noted in one patient (Case 3); a stent was retrogradely inserted via the site of occlusion at the origin of the left CCA using a 0.035 -inch guidewire through the site of arteriotomy at the internal-external carotid bifurcation. In this patient, marked topical stenosis led to occlusion, and predilation with a Mustang $6.0 \times 40$ $\mathrm{mm}$ was conducted. The topically stenotic site measured approximately 2 or $3 \mathrm{~mm}$ in length, and was sufficiently dilated by predilation. Considering the risk of recoiling, a Carotid Wallstent $10 \times 31 \mathrm{~mm}$ was inserted such that its 2or 3-mm part protruded into the arch. As distal blood flow had also diminished for a long period, the same stent $10 \times$ $24 \mathrm{~mm}$ was inserted in order for its portion to be duplicated. After treatment, TIA disappeared and there was no restenosis requiring treatment.

The 10 patients included one with Takayasu arteritis (Case 10). This patient had right CCA occlusion and marked stenosis at the origin of the left CCA. Assuming that extensive cerebral infarction may develop if the left CCA is occluded, the origin of the left external carotid artery was incised after left CEA, a 6-F sheath was inserted, and stenting for a markedly stenotic lesion of the proximal left CCA with calcification was performed. Subsequently, left external carotid artery-right CCA bypass was conducted by passing an artificial blood vessel through the anterior cervical region. In four patients in whom distal internal CAS was considered during the study period, plaque involved the distal area, but it was able to be completely removed in all patients; therefore, no patient underwent distal internal CAS.

In all patients in whom stenting was conducted, the procedure was finished and there was no procedure-related complications such as arterial dissection. No patient required blood transfusion during or after surgery. Concerning antiplatelet drugs, DAPT was performed before surgery in all patients, but there was no wound hemorrhage. After surgery, there was no whole-brain infarction, including asymptomatic infarction, myocardial infarction, or death. The mean follow-up period was 21.6 months (range: 5-31 months, median: 26 months). There was no TIA or cerebral infarction on the treated side in any patient. In one patient, restenosis required additional treatment. This patient had Takayasu arteritis (Case 10). Left CCA in-stent stenosis was observed 6 months after treatment. An additional stent was inserted into the left CCA stent through femoral artery approach. There was no treatment-related complication. In 1 patient (Case 2), an internal shunt was required. Blood was removed through a 4-F sheath inserted into the brachial artery for control angiography, and blood was supplied to the ICA side of an internal shunt via an extension tube to achieve blood flow recovery. There was no internal-shuntrelated complication.

\section{Discussion}

In the field of neurosurgery, the following surgical procedures using a hybrid operating room have been reported ${ }^{5}$ : intra-aneurysmal coil embolization after neck plastic clipping of cerebral aneurysms for complex cerebral aneurysm, ${ }^{6,7)}$ and a combination of endovascular embolization and micro-surgical treatment for cerebral arteriovenous malformations. ${ }^{8)}$ A combination of two procedures may increase the number of patients for whom treatment is indicated, improving the radical cure rate.

Many studies reported a combination of direct surgery and endovascular treatment for carotid artery stenosis using a hybrid operating room.9-17) There are some variations among these studies. Initially, there is a method to directly puncture the CCA in patients in whom accessing for CAS is difficult. ${ }^{9}$ The risk of this method may be similar to that of standard CAS; however, when the distance between the proximal end of a stent and site of puncture is near, stent deployment on the proximal side may be difficult.

On the other hand, several studies reported retrograde stenting (ipsilateral proximal endovascular intervention [IPE]) for stenotic lesions of the proximal CCA or brachiocephalic artery, ${ }^{10-17)}$ as demonstrated in our series. Many studies applied this procedure for patients with plaque continuing from the internal-external carotid bifurcation to the CCA side or in those with tandem lesions in which stenosis was separately present at the internal-external carotid bifurcation and in the proximal CCA brachiocephalic artery.

According to many studies, retrograde puncture of the external artery or CCA was performed on patients with tandem lesions free from a lesion at the internal-external carotid bifurcation. On those with tandem lesions involving a lesion at the bifurcation, CEA was initially performed, followed by stenting through the site of arteriotomy. ${ }^{11-16)}$ As an advantage of this method, vulnerable plaque or a markedly calcified lesion, which may induce embolic complications, can be accurately removed using CEA, and an atherosclerotic lesion at a site that is difficult to reach during CEA can be simultaneously treated. As three carotid branches are exposed, blood flow can be accurately blocked with a clip; therefore, debris that deviated to the arterial lumen at the 
time of stent insertion is excreted from the site of arteriotomy, and the risk of distal embolism on stenting is markedly low. In this method, the advantages of CEA and CAS are combined. Blood loss on blood excretion at the site of blockage involving debris is considered problematic, but our method to block the CCA at the distal area of a stent using a tourniquet and promote hemorrhage while controlling the volume of blood loss facilitates the instant excretion of a large volume of contaminated blood, with a minimized blood loss volume of approximately $20-30 \mathrm{~mL}$; therefore, it is useful. Although the retention of large-volume blood is transiently observed in the surgical field during CEA, the necessity of additional elimination can be reviewed by confirming the presence of debris with gauze after suction by an injector. In our series, there was no anemia requiring blood transfusion in any patient.

When indicating endovascular treatment alone for stenotic lesions of the proximal CCA or brachiocephalic artery, it is difficult to prevent distal embolism. The use of balloons that facilitate axial blockage, such as a PercuSurge GuardWire (Medtronic), makes accurate blockage of the CCA difficult due to a small diameter. Blockage of the ICA may also be appropriate, but the distance from the proximal area of the CCA is long, and the accuracy of collection with a suction catheter may be reduced when debris outflow into the blood vessel is high in the presence of large-volume plaque. For proximal blockage using a guiding catheter with a balloon, the distance to a stenotic lesion is short, making stenting difficult. Therefore, it is also difficult to establish flow reversal by proximal blockage; the prevention of distal embolism may be difficult. According to a previous study, standard trans-femoral stenting was performed after securing the CCA by incising the anterior cervix. ${ }^{10)}$ This is a method in which stenting is anterogradely conducted after blocking an area distal to the site of CCA stenosis with bulldog forceps, and a minor incision of the CCA is prepared for debris elimination. Considering the complexity of procedures to retrogradely operate catheters through the site of arteriotomy, this method may be appropriate for patients with no lesion at the internal-external carotid bifurcation. We consider the most important merit of hybrid carotid surgery to be the prevention of distal embolism by circulatory blockage at an area distal to the site of stenosis with a clip regardless of antero- or retrograde operations. In our patients, including those with asymptomatic high-signal-intensity lesions on diffusion-weighted imaging, there was no perioperative cerebral infarction.
Many studies have reported a combination of CEA and retrograde stenting (IPE) for tandem lesions involving the internal-external carotid bifurcation and proximal CCA or brachiocephalic artery. According to most reports on the perioperative results of treatment in a small number of patients, the incidence of complications was low, suggesting the safety of this combined procedure. ${ }^{1-13)}$ Bozzay et al. performed CEA+IPE on six patients, and reported that there was no perioperative cerebral infarction or death, and that there was no restenosis, cerebral infarction, myocardial infarction, or death during a follow-up of 1-36 months (mean: 12.8 months, median: 7.8 months). ${ }^{11}$ )

On the other hand, Meershoek et al. conducted CEA+IPE on 16 patients with symptomatic tandem lesions, and reported that the perioperative results of treatment were favorable, whereas the long-term results (median: 73 months, 22-85 months) included symptomatic restenosis $(n=1)$, restenosis-free ipsilateral cerebral infarction $(n=2)$, and death $(n=3)$. They emphasized that the course was not always favorable, although the detailed outcome of a spontaneous history of symptomatic tandem lesions was unclear. ${ }^{14)}$

Furthermore, several studies involving a relatively large number of patients treated by CEA+IPE reported perioperative cerebral infarction and death, suggesting that whether this treatment is indicated should be carefully evaluated. ${ }^{15,16)}$ Clouse et al. investigated the results of CEA+IPE for stenotic lesions of the proximal CCA or brachiocephalic artery in 62 patients at multiple institutions, and reported that perioperative ipsilateral cerebral infarction, hyperperfusion syndrome, and perioperative death (cerebral infarction in one patient and myocardial infarction in two patients) were noted in four $(6.5 \%)$, two, and three $(4.8 \%)$ patients, respectively. The incidence of adverse events (cerebral infarction and death) was $11.3 \%$. Furthermore, $\geq 50 \%$ restenosis was observed in 20 patients (34\%) during a mean follow-up of 6 \pm 4 years. In eight patients (14\%), additional surgery was performed (internal-external carotid bifurcation: three patients, proximal CCA: five patients). They concluded that whether CEA+IPE is indicated should be carefully evaluated in patients with symptomatic stenosis.

In this study involving a small number of patients, there was no perioperative complication. Different surgeons were responsible for CEA and CAS. The same surgeon who was experienced in each procedure performed surgery, and this may have led to the favorable results. Treatment by the same members continuously improved the procedures, reducing the operative time (Table 1). Technically, a tourniquet was used for blockage of the CCA, and debris was 
Table 1 Baseline characteristics and details of procedures that treated in hybrid operating room

\begin{tabular}{|c|c|c|c|c|c|c|c|c|c|c|}
\hline No & Age & Sex & Symptoms & Etiology & Location of lesion & $\begin{array}{c}\text { Degree of } \\
\text { stenosis (ECST) }\end{array}$ & $\begin{array}{l}\text { Length of } \\
\text { lesion }(\mathrm{mm})\end{array}$ & Procedures & $\begin{array}{c}\text { Time of } \\
\text { operation (min) }\end{array}$ & Complication \\
\hline 1 & 78 & M & TIA & Post-RT & Lt. ICA-CCA long lesion & $81 \%$ & 110 & CEA+stent & 315 & - \\
\hline 2 & 76 & $M$ & - & AS & Lt. ICA-CCA long lesion & $70 \%$ & 135 & CEA+stent & 325 & - \\
\hline 3 & 79 & M & TIA & AS & Lt. CCA CTO & - & - & CEA+stent & 320 & - \\
\hline 4 & 61 & M & - & AS & Lt. ICA-CCA long lesion & $70 \%$ & 128 & CEA+stent & 252 & - \\
\hline 5 & 62 & $\mathrm{~F}$ & Infarction & AS & Lt. ICA-CCA long lesion & $71 \%$ & 116 & CEA+stent & 210 & - \\
\hline 6 & 62 & M & Infarction & AS & Rt. ICA-CCA long lesion & $71 \%$ & 118 & CEA+stent & 210 & - \\
\hline 7 & 72 & $M$ & Infarction & AS & Lt. ICA distal & $88 \%$ & 89 & CEA only & 217 & - \\
\hline 8 & 66 & M & - & AS & Lt. ICA distal & $74 \%$ & 92 & CEA only & 185 & - \\
\hline 9 & 79 & M & - & AS & Lt. ICA-CCA long lesion & $84 \%$ & 142 & CEA+stent & 222 & - \\
\hline 10 & 43 & $\mathrm{~F}$ & - & TA & $\begin{array}{l}\text { Tandem lesion } \\
\text { Lt. Bif. \& CCA }\end{array}$ & $\begin{array}{l}\text { Bif. } 45 \% \\
\text { CCA } 82 \%\end{array}$ & $\begin{array}{l}\text { Bif. } 29 \\
\text { CCA } 42\end{array}$ & stent+bypass & 264 & - \\
\hline 11 & 72 & $\mathrm{~F}$ & - & AS & Rt. ICA-CCA long lesion & $87 \%$ & 107 & CEA only & 206 & - \\
\hline 12 & 81 & $\mathrm{~F}$ & Infarction & AS & Lt. ICA-CCA long lesion & $65 \%$ & 130 & CEA+stent & 180 & - \\
\hline 13 & 82 & $M$ & Infarction & AS & $\begin{array}{l}\text { Tandem lesion } \\
\text { Lt. ICA-Bif. \& CCA }\end{array}$ & $\begin{array}{c}\text { ICA-Bif. } 73 \% \\
\text { CCA } 65 \%\end{array}$ & $\begin{array}{l}\text { ICA-Bif. } 23 \\
\text { CCA } 34\end{array}$ & CEA+stent & 209 & - \\
\hline 14 & 69 & M & - & AS & Lt. ICA distal & $78 \%$ & 78 & CEA only & 212 & - \\
\hline 15 & 73 & $\mathrm{~F}$ & - & AS & Rt. ICA-CCA long lesion & $78 \%$ & 98 & CEA only & 183 & - \\
\hline
\end{tabular}

AS: arteriosclerosis; Bif; : bifurcation of carotid artery; CCA: common carotid artery; CEA: carotid endarterectomy; CTO: chronic total occlusion; ECST: European Carotid Surgery Trial; ICA: internal carotid artery; Post-RT: post-radiation therapy; TA: Takayasu arteritis 
Table 2 The list of devices that were used in the hybrid surgery

\begin{tabular}{|c|c|c|c|}
\hline No & Guiding & Stent & Balloon \\
\hline 1 & 8F Launcher & $\begin{array}{l}\text { Carotid Wallstent } \times 1 \\
\qquad 10 \times 31 \mathrm{~mm}\end{array}$ & $\begin{array}{l}\text { Pre: none } \\
\text { Post: Mustang } 9.0 \times 40 \mathrm{~mm}\end{array}$ \\
\hline 2 & 8F Launcher & $\begin{array}{c}\text { Carotid Wallstent } \times 2 \\
10 \times 31 \mathrm{~mm} 10 \times 24 \mathrm{~mm}\end{array}$ & $\begin{array}{l}\text { Pre: none } \\
\text { Post: Mustang } 9.0 \times 40 \mathrm{~mm}\end{array}$ \\
\hline 3 & 8F Launcher & $\begin{array}{c}\text { Carotid Wallstent } \times 2 \\
10 \times 31 \mathrm{~mm} 10 \times 24 \mathrm{~mm}\end{array}$ & $\begin{array}{l}\text { Pre: Mustang } 6.0 \times 40 \mathrm{~mm} \\
\text { Post: Mustang } 9.0 \times 40 \mathrm{~mm}\end{array}$ \\
\hline 4 & 8F Launcher & $\begin{array}{c}\text { Carotid Wallstent } \times 2 \\
10 \times 31 \mathrm{~mm} 10 \times 31 \mathrm{~mm}\end{array}$ & $\begin{array}{l}\text { Pre: none } \\
\text { Post: Mustang } 8.0 \times 40 \mathrm{~mm}\end{array}$ \\
\hline 5 & 6F Sheathless NV & Carotid Wallstent $\times 1$ & $\begin{array}{l}\text { Pre: none } \\
\text { Post: Mustang } 7.0 \times 40 \mathrm{~mm}\end{array}$ \\
\hline 6 & 8F Launcher & $\begin{array}{l}\text { Precise } \times 1 \\
10 \times 40 \mathrm{~mm}\end{array}$ & $\begin{array}{l}\text { Pre: none } \\
\text { Post: Mustang } 8.0 \times 40 \mathrm{~mm}\end{array}$ \\
\hline 9 & 6F Sheathless NV & $\begin{array}{l}\text { Precise } \times 1 \\
10 \times 40 \mathrm{~mm}\end{array}$ & $\begin{array}{l}\text { Pre: none } \\
\text { Post: Mustang } 10 \times 40 \mathrm{~mm}\end{array}$ \\
\hline 10 & $6 F$ Sheath $10 \mathrm{~cm}$ & $\begin{array}{l}\text { Assurant cobalt } \times 1 \\
10 \times 40 \mathrm{~mm}\end{array}$ & $\begin{array}{l}\text { Pre: } R \times \text { Genity } 4.0 \times 30 \mathrm{~mm} \\
\text { Post: none }\end{array}$ \\
\hline 12 & $6 F$ Sheath $10 \mathrm{~cm}$ & $\begin{array}{l}\text { Precise } \times 1 \\
10 \times 40 \mathrm{~mm}\end{array}$ & $\begin{array}{l}\text { Pre: none } \\
\text { Post: Mustang } 9.0 \times 40 \mathrm{~mm}\end{array}$ \\
\hline 13 & $6 F$ Sheath $10 \mathrm{~cm}$ & $\begin{array}{l}\text { Assurant cobalt } \times 1 \\
10 \times 40 \mathrm{~mm}\end{array}$ & $\begin{array}{l}\text { Pre: none } \\
\text { Post: none }\end{array}$ \\
\hline
\end{tabular}

Pre: pre-dilatation; Post: post-dilatation

eliminated from the site of arteriotomy while controlling the volume of blood loss. This may have reduced the incidence of embolism, leading to favorable results.

When performing hybrid surgery, it is necessary to sufficiently examine treatment procedures among occupations before surgery. The team members must be informed of the preoperative administration of antiplatelet drugs, timing of intraoperative heparin administration/its dose, anesthetic machines, surgical devices, tables for endovascular treatment, monitoring-system setting, and procedures of surgery prior to surgery. Concerning the type of stent, a balloon-dilation-type stent for the iliac artery, Assurant cobalt (Medtronic), was used to treat stenotic lesions of the proximal CCA or brachiocephalic artery not involving the CEA stump at the carotid bifurcation (Table 2) for the following reasons: positioning is easy and a post-dilation balloon is basically unnecessary. However, the use of this stent for the carotid artery is not covered by health insurance and ethical issues must be sufficiently considered.

In patients requiring stenting at the CEA stump with plaque continuing from the ICA to the CCA, a stent for CAS, Carotid Wallstent (Boston Scientific) or PRECISE (Cordis, Johnson \& Johnson, Fremont, CA, USA), was used (Table 2). When deploying the above stent, Assurant cobalt, using a balloon, excessive pressure load may be applied on the plaque-free site of arterial suture; therefore, we selected a self-dilating stent for CAS.

Furthermore, a study published in the 1990s examined revascularization under thoracotomy as a surgical procedure for stenotic lesions of the brachiocephalic artery. It was reported that although neurosurgeons were not familiar with this approach, its perioperative and long-term results were favorable, and that the incidence of complications was low, suggesting the safety of this treatment. ${ }^{17)}$ If cardiovascular surgeons' cooperation can be obtained, this treatment may be useful. However, treatment synchronous with a stenotic lesion at the internal carotid bifurcation is highly invasive, and may not be recommended with the current, widespread use of endovascular treatment procedures.

A combination of CEA and stenting through the site of arteriotomy is available for patients with chronic total occlusion (CTO) of the CCA and ICA. ${ }^{18,19)}$ We also treated a patient with symptomatic CTO of the left CCA in whom retrograde stenting was successful.

This treatment method can also be applied for trouble during CEA. In cases of ICA or CCA dissection or flap formation, this method can be used for rescue treatment by inserting a stent through the orifice of incision. If CEA for ICA stenosis, which may be technically difficult, is performed in the hybrid surgery room, many intraoperative issues can be managed, improving the safety. 
Our treatment method presented in this study has many limitations. The procedures are complex, deployment in an extensive anterior cervical surgical field under DAPT is necessary, and the risk of hemorrhagic complications must be considered, and stents or balloons that are not covered by health insurance are necessary in some cases. As the limitations of this study, the number of patients was small and the long-term outcome is unclear. Whether this treatment method is indicated should be carefully examined.

\section{Conclusion}

Hybrid surgery for carotid artery stenosis with a large volume of plaque involving an extensive area has the following limitations: the treatment setting and procedures are slightly complex, and the risk of hemorrhagic complications related to perioperative antiplatelet therapy must be considered. In the future, data should be accumulated, but hybrid surgery may be useful as a treatment option for stenotic lesions of the carotid artery that are difficult to treat by CEA or CAS alone.

\section{Disclosure Statement}

The authors declare no conflict of interest.

\section{References}

1) North American Symptomatic Carotid Endarterectomy Trial Collaborators, Barnett HJM, Taylor DW, et al: Beneficial effect of carotid endarterectomy in symptomatic patients with high-grade carotid stenosis. $N$ Engl J Med 1991; 325: 445-453.

2) Barnett HJ, Taylor DW, Eliasziw M, et al: Benefit of carotid endarterectomy in patients with symptomatic moderate or severe stenosis. North American Symptomatic Carotid Endarterectomy Trial Collaborators. N Engl J Med 1998; 339: 1415-1425.

3) Sundt TM, Whisnant JP, Houser OW, et al: Prospective study of the effectiveness and durability of carotid endarterectomy. Mayo Clin Proc 1990; 65: 625-635.

4) Brott TG, Hobson RW, Howard G, et al: Stenting versus endarterectomy for treatment of carotid-artery stenosis. $N$ Engl J Med 2010; 363: 11-23.

5) Murayama $Y$, Arakawa $H$, Ishibashi $T$, et al: Combined surgical and endovascular treatment of complex cerebrovascular diseases in the hybrid operating room. J Neurointerv Surg 2013; 5: 489-493.
6) Xin C, Li Z, Zhang J, et al: Combined surgical and endovascular treatment of complex posterior communicating artery aneurysm at one-stage in a hybrid operating room. World Neurosurg 2018; 116: 383-386.

7) Choudhri O, Mukerji N, Steinberg GK, et al: Combined endovascular and microsurgical management of complex cerebral aneurysm. Front Neurol 2013; 4: 108.

8) Grüter BE, Mendelowitsch I, Diepers M, et al: Combined endovascular and microsurgical treatment of arteriovenous malformations in the hybrid operating room. World Neurosurg 2018; 117: e204-e214.

9) Bergeron P: Direct percutaneous carotid access for carotid angioplasty and stenting. J Endovasc Ther 2015; 22: 135-138.

10) Radak D, Tanaskovic S, Sagic D, et al: A novel antegrade approach for simultaneous carotid endarterectomy and angioplasty of proximal lesions in patients with tandem stenosis of supraaortic arch vessels. Ann Vasc Surg 2017; 44: 368-374.

11) Bozzay J, Broce M, Mousa AY: Hybrid treatment of extracranial carotid artery disease. Vasc Endovascular Surg 2017; 51: 373-376.

12) Illuminati G, Pizzardi G, Pasqua R, et al: Hybrid treatment of tandem, common carotid/innominate artery and ipsilateral carotid bifurcation stenoses by simultaneous, retrograde proximal stenting and eversion carotid endarterectomy: preliminary results of a case series. Int J Surg 2018; 52: 329-333.

13) Zhang L, Xing T, Geng F, et al: Preliminary application of hybrid operation in the treatment of carotid artery stenosis in patients with complex ischemic cerebrovascular diseases. Int J Clin Exp Pathol 2014; 7: 5355-5362.

14) Meershoek AJA, Velde HM, Toorop RJ, et al: Long-term outcome of symptomatic patients undergoing hybrid revascularisation for extracranial carotid artery tandem stenosis. Eur J Vasc Endovasc Surg 2019; 57: 627-631.

15) Clouse WD, Ergul EA, Cambria RP, et al: Retrograde stenting of proximal lesions with carotid endarterectomy increases risk. J Vasc Surg 2016; 63: 1517-1523.

16) Clouse WD, Ergul EA, Wanken ZJ, et al: Risk and outcome profile of carotid endarterectomy with proximal intervention is concerning in multi-institutional assessment. $J$ Vasc Surg 2018; 68: 760-769.

17) Berguer R, Morasch MD, Kline RA: Transthoracic repair of innominate and common carotid artery disease: immediate and long-term outcome for 100 consecutive surgical reconstructions. J Vasc Surg 1998; 27: 34-41; discussion 42.

18) Jiang WJ, Liu AF, Yu W, et al: Outcomes of multimodality in situ recanalization in hybrid operating room (MIRHOR) for symptomatic chronic internal carotid artery occlusions. J Neurointerv Surg 2019; 11: 825-832.

19) Shih YT, Chen WH, Lee WL, et al: Hybrid surgery for symptomatic chronic total occlusion of carotid artery: a technical note. Neurosurgery 2013; 73: onsE117-onsE123; discussion onsE23. 